\title{
Quantitative Phase Imaging Using Deep Learning-Based Holographic Microscope
}

\author{
Jianglei Di *, Ji Wu, Kaiqiang Wang, Ju Tang, Ying Li and Jianlin Zhao* \\ MOE Key Laboratory of Material Physics and Chemistry Under Extraordinary Conditions, and Shaanxi Key Laboratory of Optical \\ Information Technology, School of Physical Science and Technology, Northwestern Polytechnical University, Xi'an, China
}

Digital holographic microscopy enables the measurement of the quantitative light field information and the visualization of transparent specimens. It can be implemented for complex amplitude imaging and thus for the investigation of biological samples including tissues, dry mass, membrane fluctuation, etc. Currently, deep learning technologies are developing rapidly and have already been applied to various important tasks in the coherent imaging. In this paper, an optimized structural convolution neural network PhaseNet is proposed for the reconstruction of digital holograms, and a deep learningbased holographic microscope using above neural network is implemented for quantitative

OPEN ACCESS

Edited by:

Peng Gao,

Xidian University, China

Reviewed by:

Wenjing Zhou,

Shanghai University, China

Yongfu Wen,

Beijing Institute of Technology, China

${ }^{*}$ Correspondence:

Jianglei $D i$

jiangleidi@nwpu.edu.cn

Jianlin Zhao

jlzhao@nwpu.edu.cn

Specialty section:

This article was submitted to

Optics and Photonics,

a section of the journal

Frontiers in Physics

Received: 09 January 2021

Accepted: 15 February 2021

Published: 22 March 2021

Citation:

Di J, Wu J, Wang K, Tang J, Li Y and Zhao J (2021) Quantitative Phase Imaging Using Deep Learning-Based

Holographic Microscope.

Front. Phys. 9:651313.

doi: 10.3389/fphy.2021.651313 phase imaging. Living mouse osteoblastic cells are quantitatively measured to demonstrate the capability and applicability of the system.

Keywords: digital holographic microscopy, digital holography, deep learning, quantitative phase imaging, convolution neural network

\section{INTRODUCTION}

Optical microscope is an effective diagnostic tool in modern healthcare which allows pathologists to clearly and qualitatively observe the details of cells and tissues, and make judgments based on experience. This technique is sufficient in most cases. However, a bright field optical microscope records the intensity information of the specimen and suffers from low contrast for transparent biological cells which presents minimal light absorption. Various labeling methods, including staining and fluorescent tagging, are designed to enhance the imaging effect of the microscope, but the dyes may cross-react with the biological processes and affect the objectivity of medical diagnosis [1]. Although phase contrast microscopy or differential interference contrast microscopy, which converts the sightless phase shifts introduced by the specimen of interest into observable intensity variations, provide an approach to survey phase specimens without labeling, they cannot provide quantitative phase information on the specimen-induced phase shifts for subsequent accurate diagnosis. Furthermore, their inherent contrast mechanism makes automated cell segmentation hardly robust.

In comparison, quantitative phase imaging techniques enable quantitative light field information and the visualization of transparent specimens [2-4]. As a typical representative of this technique, digital holographic microscopy (DHM) can be implemented for complex amplitude imaging and be used to investigate transparent specimens, such as biological samples including tissues, dry mass, membrane fluctuation, etc [5-8]. In DHM, a hologram that carries specimen information is recorded digitally first, and then the hologram is numerically reconstructed to extract the amplitude or phase of the specimens' complex field [9-13]. After that, the quantitative phase information can be converted to dry mass density of the cell with extremely high accuracy which has been demonstrated 
so far as a valuable tool in hematological or cancer diagnosis. The label-free, submicron scale sensitivity, full-field, non-destructive, real-time, quantitative and three-dimensional imaging abilities of DHM present a variety of advantages for biomedical applications, especially for live cell imaging [14-16]. Nowadays, DHM has been an important and powerful tool for medical diagnoses.

In our previous work, a common-path digital holographic microscopy based on a beam displacer unit was proposed for quantitative and dynamic phase imaging of biological cells [17]. This implementation reduces the system requirement for the light source coherence, realizes the convenient adjustment of the light beams and achieves an excellent temporal stability. However, its hologram reconstruction algorithms are often time consuming for obtaining satisfactory complex amplitude information of the specimen, which usually has certain requirements for computer hardware and need complicated tuning of user-defined parameters, such as the reconstruction distance, area and position in the frequency domain, etc. It is necessary to develop a new holographic reconstruction algorithm to improve the efficiency of common-path digital holographic microscopy.

In recent years, deep learning technology has developed rapidly, and very significant achievements have been made in areas such as autonomous driving, natural language processing, computer vision and many more. Currently, deep learning has also made remarkable achievements in computational imaging, and it has already been applied to various important tasks in coherent imaging, such as phase unwrapping [18], phase recovery [19-22], holograms reconstruction [23-27], and so on.

In this paper, an optimized structural convolution neural network PhaseNet is proposed for the reconstruction of digital holograms, and a deep learning-based holographic microscope (DLHM) using PhaseNet is implemented for quantitative phase imaging. Living mouse osteoblastic cells are quantitatively measured to demonstrate the capability and applicability of the system.

\section{METHODS}

Suppose the intensity of the recorded digital hologram in DHM is $I(x, y)$, the complex amplitude of the object light field $U(\xi, \eta)$ can be numerically reconstructed by using the scalar diffraction theory,

$$
U(\xi, \eta)=\int_{-\infty}^{\infty} \int_{-\infty}^{\infty} I(x, y) R(x, y) g(\xi, \eta, x, y) \mathrm{d} x \mathrm{~d} y,
$$

where $R(x, y)$ is the reconstruction reference light field and $g(\xi, \eta$; $x, y)$ is the impulse response function [28-30].

The hologram is usually reconstructed using convolution algorithm, corresponding reconstructed object light field $U(\xi$, $\eta)$ can be expressed as

$$
U(\xi, \eta)=\operatorname{IFFT}\{F F T\{I(x, y) R(x, y)\} \cdot \operatorname{FFT}\{g(\xi, \eta, x, y)\}\},
$$

where FFT and IFFT represent the Fourier and inverse Fourier transform operations, respectively.
And then, the intensity and phase information of the specimen can be calculated subsequently by

$$
I(\xi, \eta)=|U(\xi, \eta)|^{2}
$$

and

$$
\varphi(\xi, \eta)=\arctan \frac{\operatorname{Im}[U(\xi, \eta)]}{\operatorname{Re}[U(\xi, \eta)]}(\bmod 2 \pi)
$$

where $\operatorname{Re}$ and Im represent the real and imaginary parts of the object light field, respectively. Further by eliminating the $2 \pi$ ambiguity due to the argument operation, the true phase information of the original object wavefield can be obtained.

After the object beam passes through the biological specimen, the optical path difference $\triangle \mathrm{OPD}$ will be introduced due to the phase difference $\Delta \varphi$ and the difference of refractive index (RI) between the cells and the culture medium, which is dependent on the laser wavelength $\lambda$, the RI of the surrounding medium $n_{\text {medium }}$, the cell thickness $d$ and integral mean RI $n_{\text {specimen }}$. Therefore, the $\triangle \mathrm{OPD}$ can be calculated as

$$
\Delta \mathrm{OPD}=\left(n_{\text {specimen }}-n_{\text {medium }}\right) \cdot d=\frac{\lambda}{2 \pi} \cdot \Delta \varphi .
$$

The $\triangle \mathrm{OPD}$ is an integral effect of the optical path along the optical axis. Different parts of the cell have different RI resulting in different $\triangle \mathrm{OPD}$. Thus, in a certain sense, the $\triangle \mathrm{OPD}$ represents the thickness of the cell.

\section{DEEP LEARNING-BASED HOLOGRAPHIC MICROSCOPE}

The proposed deep learning-based holographic microscope includes a set of digital holographic microscope for the hologram recording and a deep neural network PhaseNet for the numerical reconstruction of digital holograms.

\section{Digital Holographic Microscope}

The main body of the proposed DLHM is a common-path digital holographic microscope as shown in Figure 1, which is modified based on a commercial microscope. The light source of the microscope was replaced by a fiber-coupled DPSS laser (Cobolt Samba, $532 \mathrm{~nm}$ ). Then the laser beam is focused by a lens $\mathrm{L}$ to illuminate the specimen and magnified by a $\times 20$ long working distance microscope objective MO. The transmitted light beam travels through a polarizer P1 and enters into the beam displacer $\mathrm{BD}$, in which it's separated into two orthogonally polarized beams with a small displacement. The two output beams pass through a polarizer P2 and interfere with each other at the lateral shearing region of $\mathrm{BD}$. The polarization direction of $\mathrm{P} 2$ is set at $45^{\circ}$ with the two output polarized beams, respectively. Thus, the two output beams interfere with each other in their overlap part and an off-axis digital hologram is recorded by a white-black CCD camera (target size of $5.95 \mathrm{~mm} \times$ $4.46 \mathrm{~mm}, 1,280 \times 960$ pixels, pixel size $4.65 \mu \mathrm{m} \times 4.65 \mu \mathrm{m})$. The $\mathrm{BD}$ and two polarizers are assembled together as a simple, lowcost and compact beam displacer unit, as shown in Figure 1B. In 


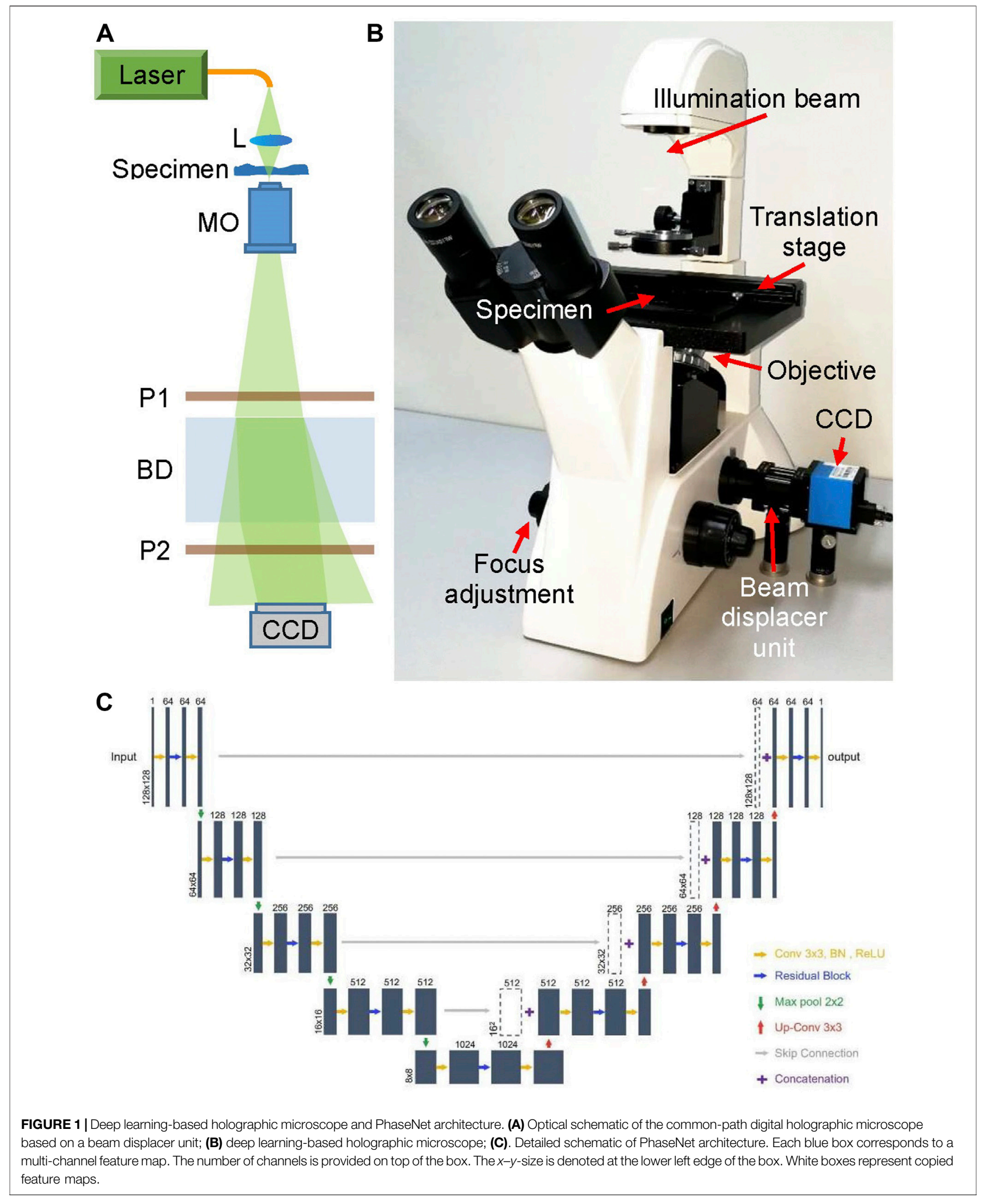


fact, benefiting from the simple optical structure and commonpath design, the commercial microscope has been improved to a digital holographic microscope for promising and potential applications in quantitative phase measurement.

\section{PhaseNet}

PhaseNet is one of the core components of data processing system of DLHM. It completes the intelligent reconstruction of the hologram and obtains the three-dimensional phase information of the specimens replacing the traditional convolution algorithm or the Fresnel transform algorithm in DHM. Figure 1C shows the detailed schematic of PhaseNet architecture which is an optimized structural CNN proposed for phase unwrapping [18]. This architecture contains three parts: down-sampling, bridge and up-sampling paths. The down-sampling path consists of five repeated use of two $3 \times$ 3 convolutions followed by a BN and a ReLU, a residual block [31], and a 2 -stride $2 \times 2 \max$ pooling operation for downsampling. The feature channels are increased by the first convolution of each repeat (first from 1 to 64 channels, the rest for double channels). By removing the max pooling from the down-sampling path, the bridge path is obtained. Each step in up-sampling consists of a transposed convolution for upsampling, a concatenation with the corresponding feature map from the down-sampling path by skip connection, two $3 \times 3$ convolutions followed by a BN and a ReLU, and a residual block between the two convolutions. The first two convolutions in each repeat decrease the number of feature channels (the last from 64 to 1 channel, the rest for halving channels). The residual blocks include two repeated use of $3 \times 3$ convolution followed by a BN and a ReLU. The skip connection is introduced to prevent the network performance degradation by summing the input and output. The downsampling path extracts and advances the features of the sinogram, while the up-sampling path reconstructs the phase from the high-level features. The channels from the first two layers are increased from 1 to 64 , which is to ensure that a sufficient number of low-level features are extracted for use in the later layers. The skip connections are added to improve the efficiency of gradient transmission.

\section{Work Procedure}

The work procedure of the proposed DLHM are as follows:

1. Hologram recording and reconstruction. Using DLHM to record off-axis digital holograms of the biological cells.

2. Phase information acquisition. Reconstructing the holograms by use of convolution algorithm to calculate the phase information of the biological cells.

3. PhaseNet training and testing. The holograms and phase results of each cell are used as input and ground truth, respectively, to train the PhaseNet. 9,000 pairs images are used for training, 1,000 pairs images for testing. Gaussian noise with random standard deviations from 0 to 25 is added into the holograms of the training dataset for better robustness. The ADAM-based optimization with an initial learning rate of 0.001 (dropping to the previous 0.75 every five epochs) is adopted to update PhaseNet's parameters. The network is trained for 200 epochs.

4. Network output obtaining. In the network training process, the PhaseNet output is calculated according to the input of the network

5. Loss function calculation. The mean squared error (MSE) of the PhaseNet output with ground truth (the phase information of biological cells) is calculated and used as the loss function. And the loss function is back-propagated through the network. 6. Quantitatively phase imaging of the biological cells. After finishing the above operations, the network training can be finally completed, and a neural network PhaseNet matching this DLHM can be obtained. Then, the digital hologram recorded by DLHM can be randomly input PhaseNet and the quantitative phase images of the specimen can be rapidly output. The network reconstruction time for a phase image is $\sim 0.014 \mathrm{~s}$.

For PhaseNet implementing, Pytorch framework based on Python 3.6.1 is used. The network training and testing are performed on a PC with Core i7-8700K CPU, using NVIDIA GeForce GTX 1080Ti GPU. The training process takes $\sim 4 \mathrm{~h}$ for 100 epochs $(\sim 10,000$ pairs images size of $128 \times 128$ pixels in a batch size of 48$)$.

\section{EXPERIMENT RESULTS AND DISCUSSIONS}

The living mouse osteoblastic cells are measured by the DLHM. These mouse osteoblastic cells IDG-SW3 are cultured in Alpha minimum essential medium (aMEM, gibco by life technologies). They stick to the bottom of the petri dish while maintaining activity and are placed on the DLHM for measurement in room temperature environment.

Figure 2 shows the numerical reconstruction results of one mouse osteoblastic cell. Figure $2 \mathrm{~A}$ is one of the recorded digital holograms of the cells in which its partially enlarged view clearly shows the interference fringes. In order to reconstruct the hologram numerically, Fourier transform is required to complete the spectral filtering. The spectrum of the hologram is shown in Figure 2B. After completing the numerical reconstruction and phase unwrapping, we can finally obtain the mouse osteoblastic cells' intensity and phase information. Figure 2C shows the quantitative two-dimensional phase map of one mouse osteoblastic cell during the mitotic phase. This phase map represents the $\triangle \mathrm{OPD}$ caused by the cell thickness, and it can also be called optical thickness of cells. In Figure 2C, some synapses are surrounding the cell. At this time, the cell is about to finish dividing and has become two cells. The biggest phase value in the central part of the two cells represents a maximum cell thickness and it's where the nucleuses are located. These are shown very clearly in the three-dimensional phase map of Figure 2D. The RI of the aMEM is $n_{\text {medium }}=1.3377$ calibrated by an Abbe refractometer, and by assuming a constant and homogeneous cellular RI $n_{\text {specimen }}=1.375$, we can estimate that a phase difference of $1 \mathrm{rad}$ corresponds to a cellular 

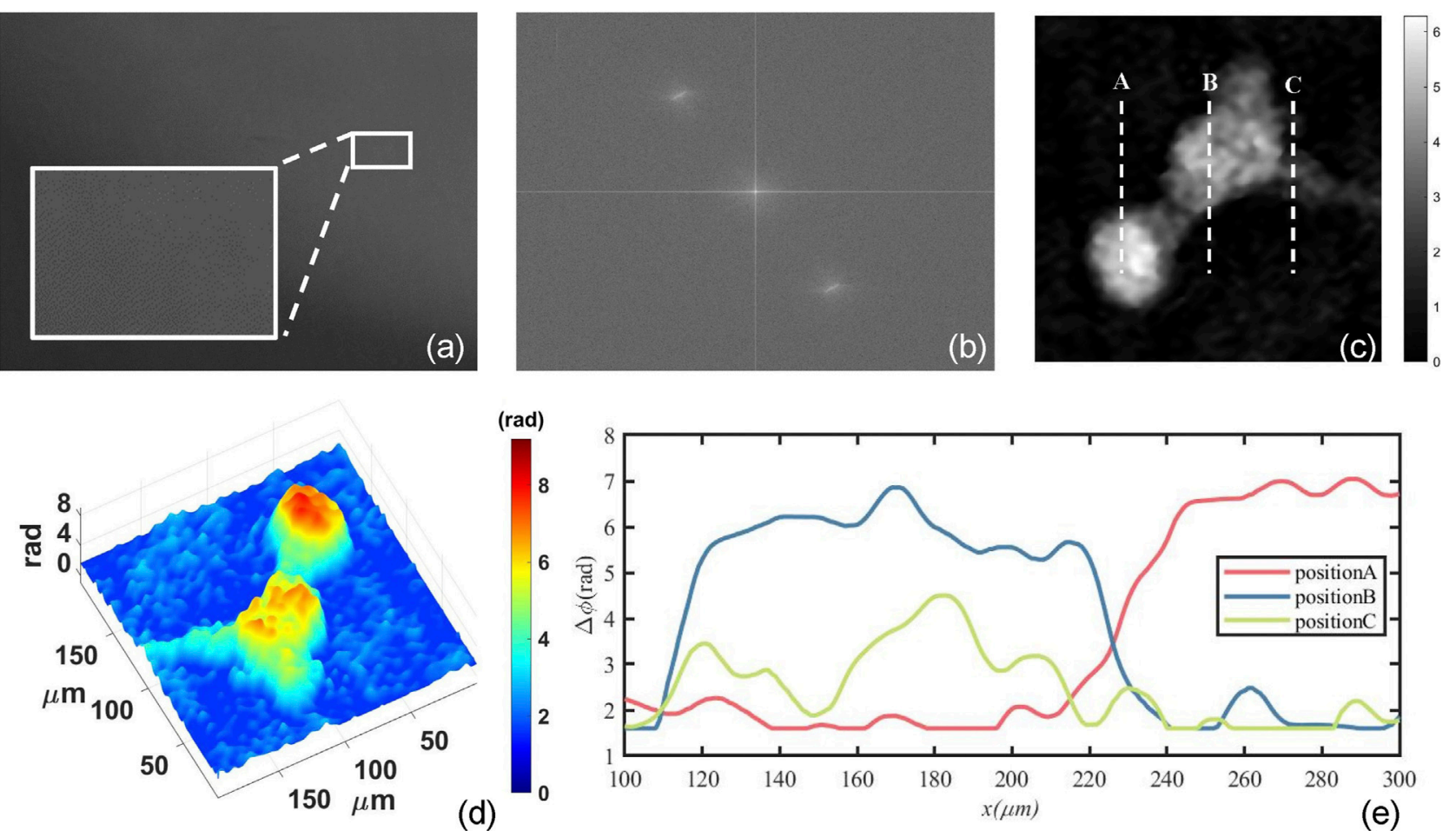

FIGURE 2 | Numerical reconstruction results of mouse osteoblastic cells by use of convolution algorithm. (A) The digital holograms of a living mouse osteoblastic cell; (B) The spectrum of the hologram; (C) The quantitative two-dimensional phase map of one mouse osteoblastic cell; (D) three-dimensional phase map; (E) The profile map along with the dash lines A, B and C in (C).
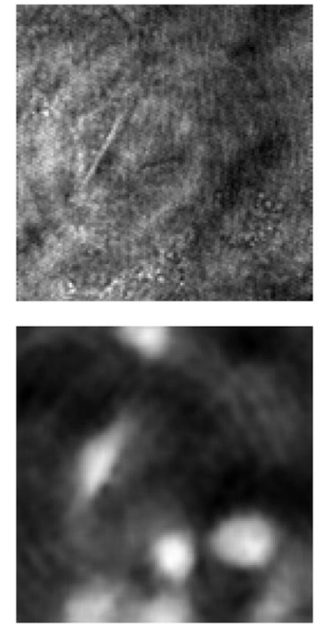
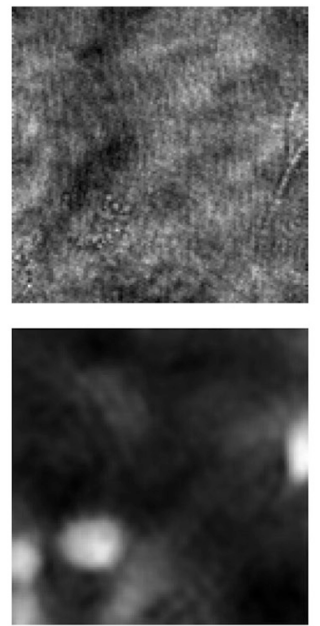
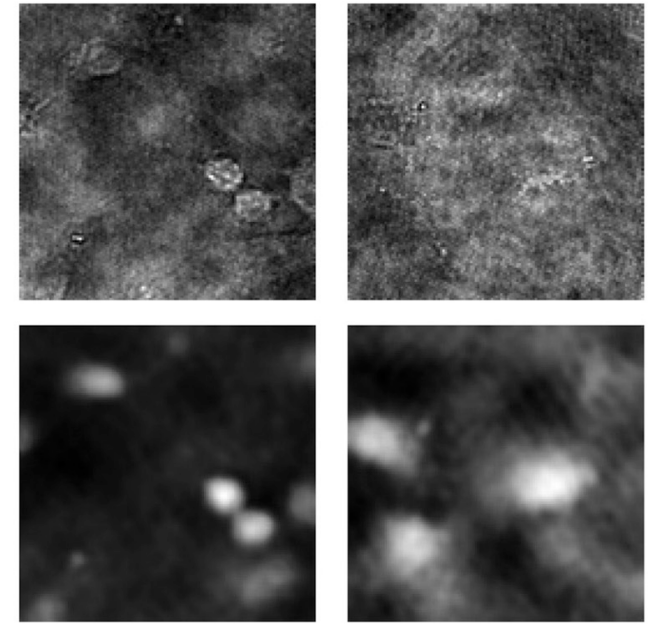
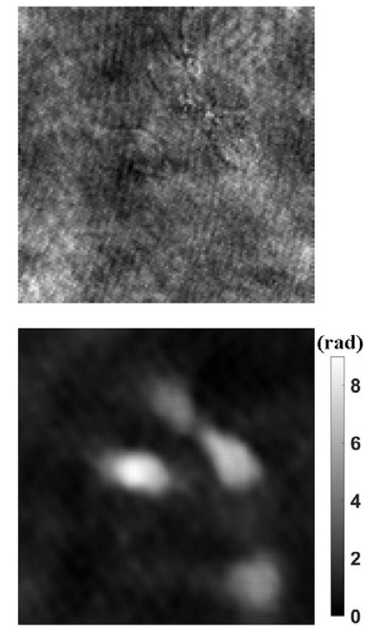

FIGURE 3 | Dataset examples. The upper part shows the holograms as input, and the lower part shows the phase results as ground truth.

thickness of $2.27 \mu \mathrm{m}$ according to Eq. 5. Figure $2 \mathrm{E}$ shows the profile map along with the dash lines A, B and C in Figure 2C. The maximum phase difference is about $7 \mathrm{rad}$ which can be translated to an optical thickness of $15.89 \mu \mathrm{m}$.

1990 holograms of mouse osteoblastic cells are taken with DLHM. Then the phase images are recovered by traditional convolution algorithm. In order to improve the generalization ability of the neural network, data augmentation is a standard method. The dataset including 1990 holograms and corresponding phase images is expanded to 10,000 by flipping, rotating, etc. After that, the holograms and phase images of each cell are used as input and ground truth, respectively. Figure 3 

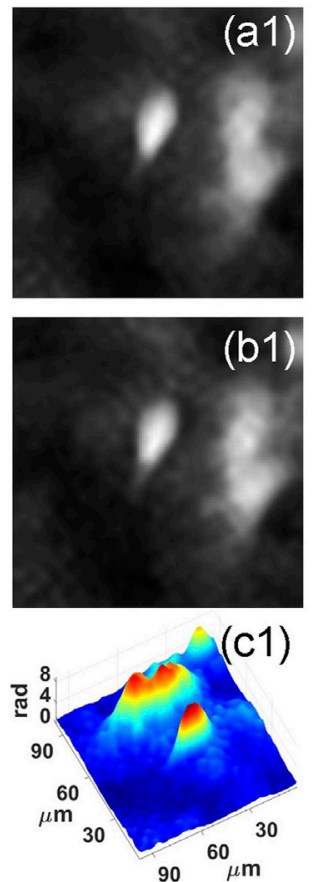

(d1)

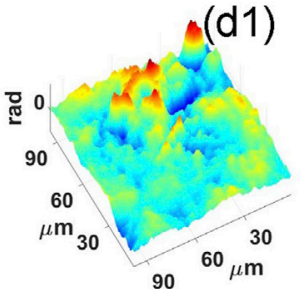

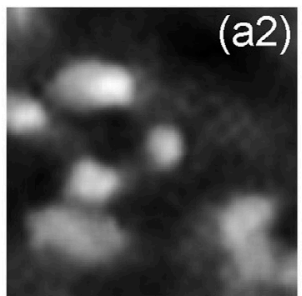
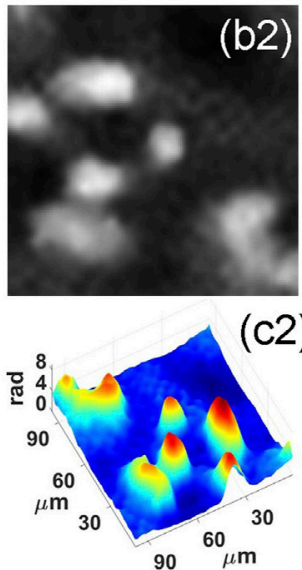

(d2)

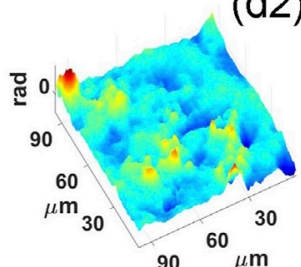

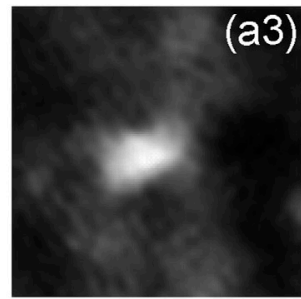
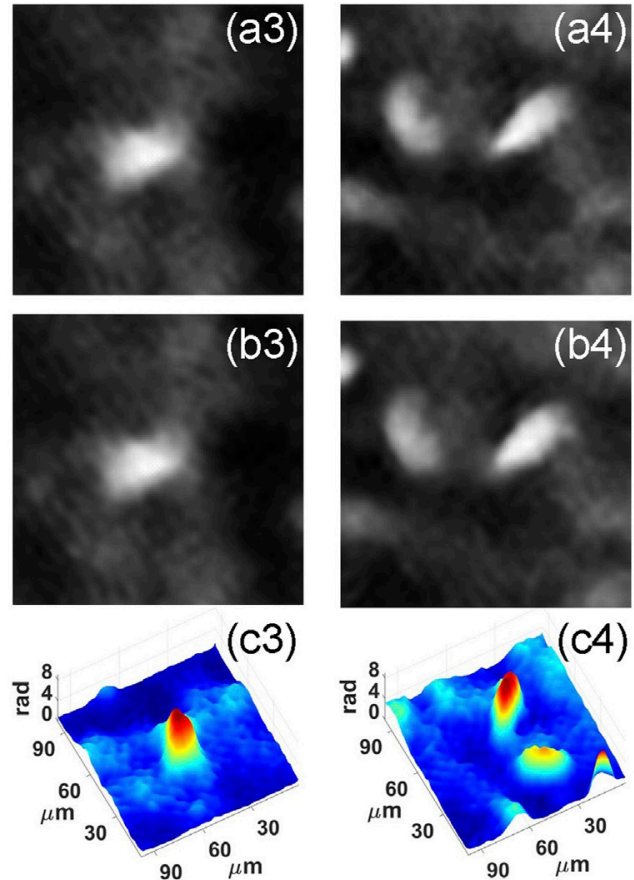

(d3)

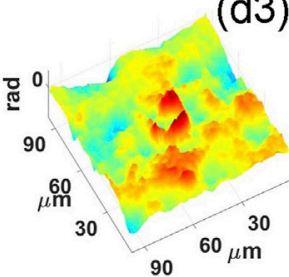

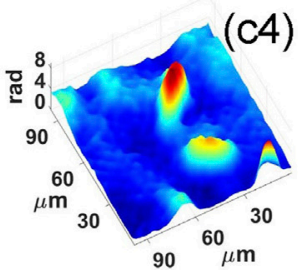

(d4)

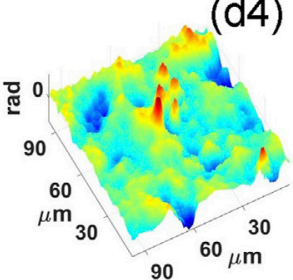

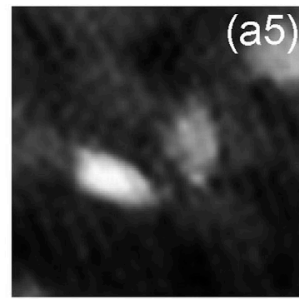
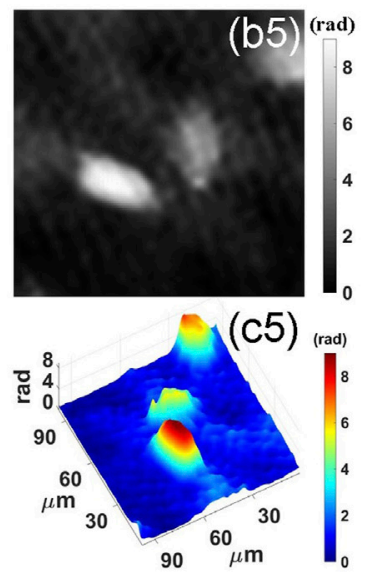

(d5)

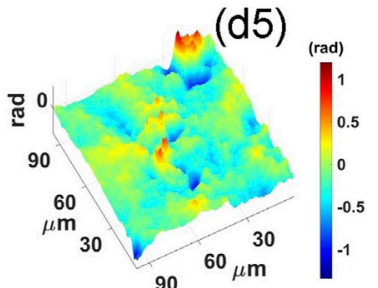

FIGURE 4 | Neural network reconstruction results. (A) Ground truth; (B) PhaseNet reconstruction result; (C) Three-dimensional image of PhaseNet reconstruction result; (D) Three-dimensional image of error maps between Ground truth and PhaseNet reconstruction result.

shows part of the dataset. Among all the data, $90 \%$ are used for training, $10 \%$ for testing. Then we can use the prepared dataset to train the neural network PhaseNet.

As the training progresses, the MSE of the ground truth and the output are back-propagated to the network, and parameters such as weights are updated by gradient descent. After 100 epoch training, the network reaches the convergence state. In the beginning, the loss function drops the fastest, as the epoch progresses, it becomes slower and slower, and the speed is close to zero at 100 epochs.

After training, we feed the holograms in the test set to PhaseNet, and the corresponding phase results are quickly reconstructed. Part of the results is visualized in Figure 4. Figures $4 \mathbf{A}, \mathbf{B}$ are the ground truth and corresponding reconstruction results, respectively. From the twodimensional phase images of the cell, we can see that the results of the network reconstruction are very close to the ground truth. More quantitatively, we calculated its structural similarity index measure (SSIM), which is used for measuring the similarity between two images and is a perception-based model that considers image degradation as perceived change in structural information, while also incorporating important perceptual phenomena, including both luminance masking and contrast masking terms. Finally, the SSIM of the phase results obtained using PhaseNet can reach 0.9404. This shows that PhaseNet can replace traditional algorithms to achieve the numerical reconstruction of holograms. Figure 4C shows the three-dimensional phase images of the cells obtained by PhaseNet.

After completing the network training, the deep learningbased holographic microscope is feasible for quantitative phase measurement of living biological cells. It can completely replace the traditional digital holographic microscope for label-free cell imaging. At the same time, due to the use of neural networks, the acquisition of three-dimensional information of specimens can be completed more quickly.

In conclusion, we proposed PhaseNet for the reconstruction of digital holograms, based on which the DLHM is implemented for quantitative phase imaging of biological specimens. In order to verify the capability and applicability of DLHM, we used the living mouse osteoblastic cells as samples to generate dataset and train PhaseNet. The testing results show that the average SSIM index of DLHM can reach 0.9404 . 


\section{DATA AVAILABILITY STATEMENT}

The raw data supporting the conclusions of this article will be made available by the authors, without undue reservation.

\section{AUTHOR CONTRIBUTIONS}

JD and JZ conceived and supervised the project. YL and KW performed experiments and data analysis. KW, JW and JT

\section{REFERENCES}

1. Park Y, Depeursinge C, Popescu G. Quantitative phase imaging in biomedicine. Nat Photon (2020) 12:578-89. doi:10.1364/cleo_si.2012.ctu3j.5

2. Ahmad A, Dubey V, Singh G, Singh V, Mehta DS. Quantitative phase imaging of biological cells using spatially low and temporally high coherent light source. Opt Lett (2016) 41:1554-7. doi:10.1364/ol.41.001554

3. Creath K, Goldstein G. Dynamic quantitative phase imaging for biological objects using a pixelated phase mask. Biomed Opt Express (2012) 3:2866-80. doi:10.1364/boe.3.002866

4. Popescu G, Quantitative phase imaging of nanoscale cell structure and dynamics, BP Jena. Quantitative phase imaging of nanoscale cell structure and dynamics. Methods Cel Biol (2008) 90:87. doi:10.1016/S0091-679X(08) 00805-4

5. Fan X, Healy JJ, O'Dwyer K, Hennelly BM. Label-free color staining of quantitative phase images of biological cells by simulated Rheinberg illumination. Appl Opt (2019) 58:3104-14. doi:10.1364/ao.58.003104

6. Doblas A, Sanchez-Ortiga E, Martinez-Corral M, Saavedra G, Garcia-Sucerquia J. Accurate single-shot quantitative phase imaging of biological specimens with telecentric digital holographic microscopy. J Biomed Opt (2014) 19:29. doi:10. 1117/1.jbo.19.4.046022

7. Marquet P, Rappaz B, Magistretti PJ, Cuche E, Emery Y, Colomb T, et al. Digital holographic microscopy: a noninvasive contrast imaging technique allowing quantitative visualization of living cells with subwavelength axial accuracy. Opt Lett (2005) 30:468-70. doi:10.1364/ol.30.000468

8. Rong L, Latychevskaia T, Chen C, Wang D, Yu Z, Zhou X, et al. Terahertz inline digital holography of human hepatocellular carcinoma tissue. Scientific Rep (2015) 5:33. doi:10.1038/srep08445

9. Gao P, Yao B, Min J, Guo R, Ma B, Zheng J, et al. Autofocusing of digital holographic microscopy based on off-axis illuminations. Opt Lett (2012) 37: 3630-2. doi:10.1364/ol.37.003630

10. Ma J, Yuan C, Situ G, Pedrini G, Osten W. Resolution enhancement in digital holographic microscopy with structured illumination. Chin Opt Lett (2013) 11: 124. doi:10.3788/col201311.090901

11. Guo R, Yao B, Gao P, Min J, Zhou M, Han J, et al. Off-axis digital holographic microscopy with LED illumination based on polarization filtering. Appl Opt (2013) 52:8233-8. doi:10.1364/ao.52.008233

12. Gao P, Pedrini G, Osten W. Structured illumination for resolution enhancement and autofocusing in digital holographic microscopy. Opt Lett (2013) 38:1328-30. doi:10.1364/ol.38.001328

13. Weng J, Zhong J, Hu C. Digital reconstruction based on angular spectrum diffraction with the ridge of wavelet transform in holographic phase-contrast microscopy. Opt Express (2008) 16:21971-81. doi:10.1364/oe.16.021971

14. Di J, Li Y, Xie M, Zhang J, Ma C, Xi T, et al. Dual-wavelength common-path digital holographic microscopy for quantitative phase imaging based on lateral shearing interferometry. Appl Opt (2016) 55:7287-93. doi:10.1364/ao.55. 007287

15. Min J, Yfao B, Ketelhut S, Engwer C, Greve B, Kemper B. Simple and fast spectral domain algorithm for quantitative phase imaging of living cells with digital holographic microscopy. Opt Lett (2017) 42:227-30. doi:10.1364/ol.42. 000227

16. Zhong Z, Zhao HJ, Cao LC, Shan MG, Liu B, Lu WL, et al. Automatic cross filtering for off-axis digital holographic microscopy. Results Phys (2020) 16:6. doi:10.1016/j.rinp.2019.102910 contributed to data analysis. KW, JW and JD wrote the draft of the manuscript; All the authors edited the manuscript.

\section{FUNDING}

This work was supported by National Natural Science Foundation of China (NSFC) $(62075183,61927810)$ and NSAF (U1730137).

17. Di J, Li Y, Wang K, Zhao J. Quantitative and dynamic phase imaging of biological cells by the use of the digital holographic microscopy based on a beam displacer unit. IEEE Photon J (2018) 10(4):6900510. doi:10.1109/jphot. 2018.2839878

18. Wang K, Li Y, Kemao Q, Di J, Zhao J. One-step robust deep learning phase unwrapping. Opt Express (2019) 27:15100-15. doi:10.1364/oe.27.015100

19. Rivenson Y, Zhang Y, Gnaydin H, Teng D, Ozcan A. Phase recovery and holographic image reconstruction using deep learning in neural networks. Light-Science Appl (2018) 7:127. doi:10.1038/lsa.2017.141

20. Wang K, Di J, Li Y, Ren Z, Kemao QJ. Transport of intensity equation from a single intensity image via deep learning. Opt Lasers Eng (2020) 134:106233. doi:10.1016/j.optlaseng.2020.106233

21. Zhao F, Bian Y, Wang H, Lyu M, Pedrini G, Osten W, et al. Phase imaging with an untrained neural network. Light-Science Appl (2020) 9:127. doi:10.1038/ s41377-020-0302-3

22. Zhou W-J, Guan X, Liu F, Yu Y, Zhang H, Poon T-C, et al. Phase retrieval based on transport of intensity and digital holography. Appl Opt (2018) 57: A229-A234. doi:10.1364/ao.57.00a229

23. Rivenson Y, Wu Y, Ozcan A. Deep learning in holography and coherent imaging. Light-Science Appl (2019) 8:44. doi:10.1038/s41377-019-0196-0

24. Wang K, Dou J, Kemao Q, Di J, Zhao J. Y-Net: a one-to-two deep learning framework for digital holographic reconstruction. Opt Lett (2019) 44:4765-8. doi:10.1364/ol.44.004765

25. Wang K, Kemao Q, Di J, Zhao J. Y4-Net: a deep learning solution to one-shot dual-wavelength digital holographic reconstruction. Opt Lett (2020) 45: 4220-3. doi:10.1364/ol.395445

26. Yan K, Yu Y, Huang C, Sui L, Qian K, Asundi A. Fringe pattern denoising based on deep learning. Opt Commun (2019) 437:148-52. doi:10.1016/j.optcom.2018.12.058

27. Ren Z, So HK-H, Lam EY. Fringe pattern improvement and super-resolution using deep learning in digital holography. IEEE Trans Ind Inf (2019) 15: 6179-86. doi:10.1109/tii.2019.2913853

28. Di J, Zhao J, Sun W, Jiang H, Yan X. Phase aberration compensation of digital holographic microscopy based on least squares surface fitting. Opt Commun (2009) 282(19):3873-7. doi:10.1016/j.optcom.2009.06.049

29. Sun W, Zhao J, Di J, Wang Q, Wang L. Real-time visualization of Karman vortex street in water flow field by using digital holography. Opt Express (2009) 17(22):20342-8. doi:10.1364/oe.17.020342

30. Di J, Yu Y, Wang Z, Qu W, Cheng CY, Zhao J. Quantitative measurement of thermal lensing in diode-side-pumped Nd:YAG laser by use of digital holographic interferometry. Opt Express (2016) 24(25):28185-93. doi:10. 1364/oe.24.028185

31. He K, Zhang X, Ren S, Sun J. Deep residual learning for image recognition. IEEE Conf. Comput. Vis. Pattern Recognit (2016) 14:770-8. doi:10.1109/cvpr.2016.90

Conflict of Interest: The authors declare that the research was conducted in the absence of any commercial or financial relationships that could be construed as a potential conflict of interest.

Copyright (c) $2021 \mathrm{Di}, \mathrm{Wu}$, Wang, Tang, Li and Zhao. This is an open-access article distributed under the terms of the Creative Commons Attribution License (CC BY). The use, distribution or reproduction in other forums is permitted, provided the original author(s) and the copyright owner(s) are credited and that the original publication in this journal is cited, in accordance with accepted academic practice. No use, distribution or reproduction is permitted which does not comply with these terms. 